\title{
Medically unexplained visual loss in children and young people: an observational single site study of incidence and outcomes
}

${ }^{1} \mathrm{NIHR}$ Biomedical Research Centre at Moorfields Eye Hospital and UCL Institute of Ophthalmology,

London, UK

${ }^{2}$ Eye Center, Medical Center, University of Freiburg, Freiburg, Germany

${ }^{3}$ Great Ormond Street Hospital for Children NHS Foundation Trust, University College London, Institute of Child Health, London, UK

Correspondence:

AH Dahlmann-Noor, NIHR Biomedical Research Centre at Moorfields Eye Hospital and UCL Institute of Ophthalmology, 162 City Road, London EC1V 2PD, UK

Tel: +44 (0)20 7566 2013;

Fax: +44 (0)20 75662016.

E-mail: annegret.dahlmannnoor@moorfields.nhs.uk

Received: 24 November 2016

Accepted in revised form: 13 January 2017

Published online: 10 March 2017

\begin{abstract}
Purpose To determine the incidence of medically unexplained visual loss (MUVL) in children in an open access children's eye casualty.

Patients and methods We collated demographic and clinical data of consecutive patients younger than 16 years who presented to the children's eye casualty at Moorfields Eye Hospital over a 12-month period and were diagnosed with MUVL or suspected MUVL. We reviewed the clinical records at least 3 months after initial presentation. We calculated the incidence using the number of 'new patient' attendances over the same period as denominator $(n=2397)$. We used descriptive analysis. Main outcome measures: number of patients diagnosed with MUVL, proportion of patients with a history of or present psychological problems, recovery rate, and improvement in visual acuity.
\end{abstract}

Results We identified 85 cases of MUVL (54 females; median age: 9 years (IQR 7-12)). The median duration of follow-up was 1.2 months (IQR 0-4.3). The estimated annual incidence was $3.5 \%$ (95\% confidence interval $2.9-4.4 \%)$. Thirty-three per cent of children had a history of psychiatric disorders, reported a stressful life event, or showed signs of psychiatric disorder at the time of first presentation. The recovery rate was $25 \%$. Median improvement in best-corrected visual acuity from presentation to last appointment was 0.22 (IQR 0.06-0.43) logMAR.

Conclusion The incidence of MUVL is higher and the rate of resolution lower than previously reported. MUVL may be associated with mental health problems. We recommend screening for psychological problems to facilitate access to psychological treatment.
MC Daniel 1,2, A Coughtrey ${ }^{3}$, I Heyman ${ }^{3}$ and AH Dahlmann-Noor ${ }^{1}$

Eye (2017) 31, 1068-1073; doi:10.1038/eye.2017.37; published online 10 March 2017

\section{Introduction}

Medically unexplained visual loss (MUVL) describes visual loss or visual symptoms in the absence of any medically detectable eye, visual pathway, or brain condition. It is classified as a conversion disorder, in DSM-5, that is, a functional neurological symptom disorder resulting in loss of function. As with other medically unexplained symptoms, there is no universally accepted definition. ${ }^{1}$ A number of different terms are used to describe the condition, and terminology has evolved over time (MUVL, non-organic visual loss, functional visual loss, hysterical visual loss, malingering, non-physiologic visual loss, factitious visual loss, psychogenic visual loss, hypochondriasis, and conversion disorder of vision). ${ }^{2-5}$ We will use the term medically unexplained vision loss throughout this manuscript as this is the term families have told us is most acceptable as it makes no assumptions about cause.

In children, MUVL is not uncommon. The reported prevalence ranges from 1 to $9 \%{ }^{6-8}$ The incidence of MUVL has been estimated at $1-1.75 \%{ }^{9,10}$ As in adults with MUVL ${ }^{11}$ and other medically unexplained symptoms, ${ }^{12}$ socioeconomic factors may also contribute to MUVL in children.

All previous studies indicate that girls are more commonly affected than boys. ${ }^{9,13-15}$ The reported mean age at presentation ranges from 9.0 to 13.4 years, but younger children can also be affected. ${ }^{9,10,13-18}$ The most common complaints are deterioration of visual acuity, visual field defects, and double vision. $., 9,14,15$ In the majority of cases, both eyes are 
affected. ${ }^{9,13-15}$ Other symptoms are photopsia (perception of flashes of light that are usually brief and intermittent), perception of phosphenes (light perceptions of any colour or shape other than intermittent flashes that are not induced by light entering the eye), photophobia (light hypersensitivity), dyschromatopsia (altered perception of colours), amblyopia, voluntary nystagmus, accommodation weakness, ptosis, blepharospasm, and painful eyes. 3,5,14,15,19 Some children have a history of previous eye diseases and treatment. ${ }^{13,20}$ MUVL in the presence of known eye diseases, and/or nonocular conditions such as asthma, autoimmune diseases and accidental ${ }^{13,21}$ or surgical trauma is referred to as functional overlay. $^{20}$

Children with MUVL are more likely to also report other medically unexplained physical symptoms such as headaches and abdominal or limb pain. ${ }^{14}$ MUVL is also associated with factors similar to those underlying other medically unexplained physical symptoms. For example, 40-90\% 9,19,22 of children with MUVL also report psychological stressors such as family problems, problems at school, or bullying. ${ }^{10,19,23}$ High rates of mental health problems have been reported in adults with MUVL, ${ }^{14}$ and some research has indicated that young people with MUVL are more likely to report symptoms such as depression and attention deficit hyperactivity disorder. ${ }^{16,24-27}$ As with other medically unexplained symptoms, there are likely to be multiple interacting causal factors, and the presence of comorbid mental health disorders does not suggest that symptoms are 'all in the mind'. In other medically unexplained symptoms (eg, headache, stomach pains, and so on), as many as $30-50 \%$ of children have associated mental health disorders. ${ }^{28}$ Screening for and detecting mental health problems in children with MUVL may facilitate access to appropriate services. 2,4,14,15

The rate of spontaneous resolution of MUVL in children has been reported to be high, particularly in studies with long follow-up data, ranging from $37 \%$ at 12 months ${ }^{10}$ to $100 \%{ }^{9}$ (unknown duration of follow-up); the management of MUVL therefore often focuses on providing reassurance to the child and family that the visual prognosis is excellent.

There are no management recommendations for ophthalmologists, beyond the establishment of the diagnosis. To establish current practice and outcomes and to facilitate service planning and the development of future research projects, we carried out a retrospective observational study to describe incidence, clinical characteristics of patients, current diagnostic workup, and outcomes of MUVL in children.

\section{Subjects and methods}

This service evaluation had Trust approval (CA16/ ONSP/91). A research fellow (MCD) collated demographic and clinical data of consecutive patients younger than 16 years who presented to the children's eye casualty at Moorfields Eye Hospital over a 12-month period and were diagnosed with MUVL or suspected MUVL. The research fellow reviewed clinical records at least 3 months after the initial presentation. All information was gathered from the clinical notes. To keep bias to a minimum, the same person collected data from all the files. We calculated the incidence using the number of 'new patient' attendances over the same period as denominator $(n=2397)$. Patients were included into the study if a diagnosis of 'MUVL' or 'functional visual loss' was documented in the medical notes and was not revised over subsequent clinic visits. We recorded any history of previous eye problems that had occurred at least 4 weeks before the presentation that led to a diagnosis of MUVL and could therefore be reasonably assumed to be unrelated. Children were considered as having fully recovered if they felt the eye problems had completely resolved and visual acuity was at least 0.1 $\log$ MAR in the initially affected eye. In cases where visual acuity at first presentation could not be determined in $\log$ MAR values ('hand movements' or 'perception of light'), we did not quantify the change in vision between visits. The main outcome measures were the number of patients diagnosed with MUVL, the proportion of patients with a history of or present psychological problems, the recovery rate, and the improvement in visual acuity. All data were analysed using descriptive statistics. We adjusted denominators for missing data. To address loss to follow-up, the recovery rate was calculated separately for children with a follow-up of 3 months or less and for the subgroup of children who had a follow-up of 3 months or longer.

\section{Results}

\section{Incidence}

We identified 88 cases of suspected MUVL. Three children were subsequently found to have isolated optic disc atrophy, macular dystrophy, or optic neuropathy, confirmed by abnormal electrophysiological findings. These children were excluded from the analysis. Eightyfive cases were included in the analysis. We estimated the annual incidence in our setting to be 3.5\% (95\% confidence interval $2.85-4.35 \%$ ). The number of children diagnosed with MUVL peaked during the winter months (Figure 1).

\section{Patient characteristics}

The median age at presentation was 9 years (IQR 7-12). Fifty-four patients were girls. The median number of 


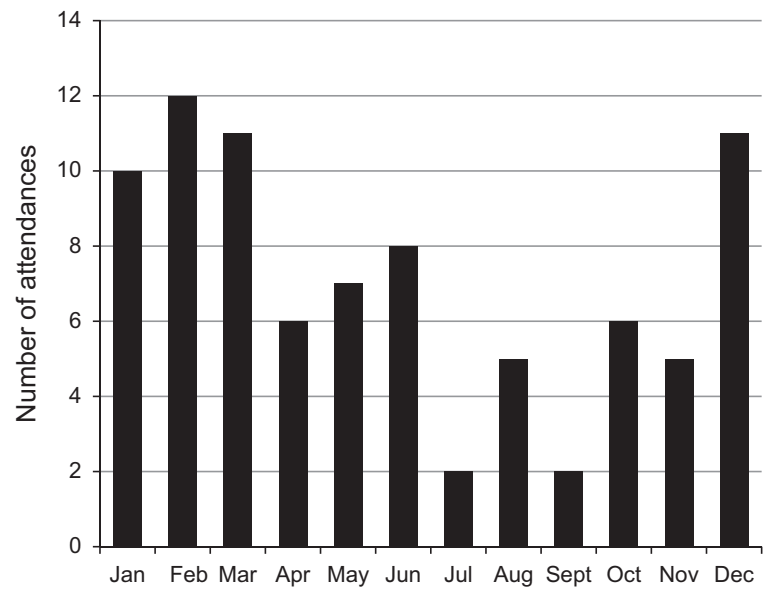

Figure 1 Seasonal variation of the number of children diagnosed with MUVL. Peak in the winter months.

appointments was 2 (IQR 1-3). Twenty-eight children only attended once. The median time from onset of symptoms until initial presentation was 1 week (IQR 0.14-2); the median duration of follow-up was 1.2 months (IQR 0-4.3) (Table 1).

\section{Diagnostic workup and findings}

There was considerable variability in diagnostic investigations (Table 1). Thirty-one per cent of all children were diagnosed with orthoptic abnormalities or refractive errors, and $12 \%$ showed abnormal visual field test results. Fifty-seven per cent had a previous history of contact with eye health professionals for glasses or surgical procedures.

None of the children seen during this period were referred for psychological assessment or intervention.

\section{Patient history and presentation}

Sixty-four per cent of all children had bilateral symptoms. Thirty-six per cent had a history of eye problems or ocular surgery. Forty-one per cent had glasses at first presentation. The most common complaints were deterioration of visual acuity (68\%), painful eyes (24\%), photopsia or perception of phosphenes (19\%), and diplopia (19\%). Complete loss of vision $(13 \%)$, photophobia ( $9 \%)$, visual field loss $(7 \%)$, and swollen lids (7\%) were less common.

Ocular symptoms were associated with non-ocular symptoms in $35 \%$ of all cases, headache being the most common complaint (93\%).

Forty-eight per cent of all children had non-ocular health problems such as allergies, asthma, and hypothyroidism. Rare diagnoses were complex regional pain syndrome, lactose intolerance, Marfan syndrome,
Table 1 Incidence, clinical workup, and demographical and clinical characteristics of children diagnosed with MUVL

Number of children diagnosed with MUVL

85

Age (years)

Number of appointments

Duration of follow-up (days)

$\begin{array}{cl}\text { Median } & I Q R \\ 9 & 7-12 \\ 2 & 1-3 \\ 35 & 0-130\end{array}$

Ratio (female : male)

1.7

Investigations

Orthoptic assessment

Refraction

Perimetry

Visual electrodiagnostics

Optical coherence tomography

Referral for paediatric assessment

Neuroimaging

$\begin{array}{rr} & \% \\ 7 & 65 \\ 7 & 53 \\ 4 & 27 \\ 1 & 24 \\ 3 & 15 \\ 2 & 14 \\ 5 & 6\end{array}$

Psychological characteristics

History of psychiatric problems

External stressors

Clinical signs of depression

Full recovery at last follow-up Within 3 months

After at least 3 months follow-up

Incidence

migraine, and thalassaemia. A brief behavioural and emotional symptom history, and a history of previous clinical service use were taken as is usual in any paediatric consultation. Thirty-three per cent of all children had a history of psychiatric disorders or showed signs of psychiatric disorder at the time of first presentation. One child currently reported current clinical levels of depression (under psychiatric care), three children had a history of psychiatric problems, but no longer showed symptoms at the time of presentation, and $28 \%$ reported stressful live events. Twenty-four per cent reported recent injuries.

\section{Clinical course and resolution of symptoms}

At last follow-up, 21 children (25\%) had fully and 12 $(14 \%)$ had partially recovered (resolution rate at 3 months after first presentation: $13 \%$ and resolution rate after at least 3 months of follow-up: 34\%). The median improvement in best-corrected visual acuity (worse affected eye) was $0.22 \log$ MAR (IQR 0.06-0.43). When tested for visual acuity, four children claimed not to be able to see anything or to perceive light or hand movements only; we excluded these from the analysis. Three of these children had normal visual acuities at the 
last follow-up. One child did not report any clinically significant improvement of visual acuity.

\section{Discussion}

Our principal finding of a 3.5\% incidence of MUVL in children and young people attending a specialist ophthalmological hospital is two to three times higher than previously reported. ${ }^{10,11}$ Our study also challenges the commonly held belief that-in children-MUVL has a high rate of spontaneous resolution. We report here that 3 months after presentation, $87 \%$ of those children who had at least 3 months of follow-up still experienced vision problems.

The high incidence of MUVL in this study may be explained in part by the setting, a walk-in clinic in a specialist ophthalmological hospital providing tertiary care level workup for patients who often have not consulted their general practitioner or local optometrist before attending our service. We observed that a high proportion of children with MUVL had previous experience with eye care providers; this may have raised their awareness of the possibility of having eye problems and direct access to eye care providers. The number of children diagnosed with MUVL peaked during the winter months, indicating possible seasonal variation. However, our sample size is small and limited to one calendar year only. In addition, we did not systematically ask children about any seasonal stressors/relieving factors (eg, school examinations/school holidays). Further research is required to establish any seasonal variation in the incidence of MUVL.

MUVL may have commonalities with other medically unexplained symptoms such as headaches, abdominal pain, and non-epileptic seizures, including co-morbidity with common mental health disorders including anxiety, depression, and behavioural problems. ${ }^{28}$ Currently, there is little known about the mental health of young people with MUVL, and there is no routine screening, established referral pathways or guidelines for the detection and management of mental health difficulties associated with MUVL. It has been suggested that an absence of mental health disorders may be associated with faster resolution of symptoms, ${ }^{24}$ though this finding is controversial. ${ }^{14,19}$ Though psychiatric consultation has not yet been shown to improve final visual outcome, ${ }^{7}$ patients may benefit from addressing psychological issues. ${ }^{13}$ Cognitive behavioural and whole-system approaches to the management of other medically unexplained symptoms and associated mental health difficulties are successful for the management for both adult patients and children and young people. ${ }^{25-27,29,30}$ Screening for psychiatric comorbidity in MUVL in young people will allow early detection of emotional and behavioural problems, and facilitate access to evidence-based psychological therapies. Therefore, a comprehensive multidisciplinary assessment of these children is likely to include, in addition to the ophthalmological and medical history, a mental health review, family history, and social and educational history.

The low rate of recovery in this study compared with other publications $\left(93^{14}-100 \%{ }^{9}\right)$ may in part be explained by the lack of a standardised definition of 'complete resolution' and by the relatively short follow-up in our study. Ophthalmologists are often satisfied when good vision can be demonstrated, and limit management to providing a 'strong dose of reassurance' that symptoms will resolve. ${ }^{7-9,13}$ Some discuss psychological aspects with the family and the general practitioner. ${ }^{15}$ Few refer children for neuropsychological evaluation. ${ }^{5}$ In other medically unexplained conditions, the presence of an unrecognised co-morbid mental health problem can impact negatively on the symptom trajectory. ${ }^{28,31}$ In recent years, there has been an emphasis on integrating mental and physical healthcare, therefore it is necessary to ensure that young people with MUVL are referred to appropriate evidence-based services for treatment if a psychiatric co-morbidity is identified.

Limitations of our work include data collection at a single site in a highly urbanised area and the relatively short follow-up duration. The present study does not allow conclusions on the long-term course of MUVL. Longitudinal studies with a longer follow-up duration could provide valuable information on the fluctuation of symptoms and the likelihood of relapses, and/or the simultaneous or delayed manifestation of other types of medically unexplained symptoms. However, our setting caters for a multi-ethnic urban population and we expect our findings could be replicated in similar settings. A further limitation is the current lack of a 'positive diagnosis' and a lack of consensus in terminology. A recent qualitative study of non-epileptic seizures highlighted the importance of families and young people having ownership over the terminology used to describe their condition, ${ }^{22,33}$ and the field is likely to be advanced through qualitative studies to explore and examine the experiences of young people with MUVL.

To achieve optimum and rapid recovery in paediatric MUVL, it is likely that integrated ophthalmological and mental health assessment and treatment will be needed. The low rate of full recovery of MUVL with ophthalmological approaches alone suggests that additional interventions may be needed. Identifying, understanding, and alleviating psychosocial stressors may be important as they may be precipitants or causes of MUVL. In addition, establishing rates of psychiatric comorbidity (eg, anxiety, depression, and so on) in these 
children will improve understanding of mechanisms and identify additional treatment targets.

Summary

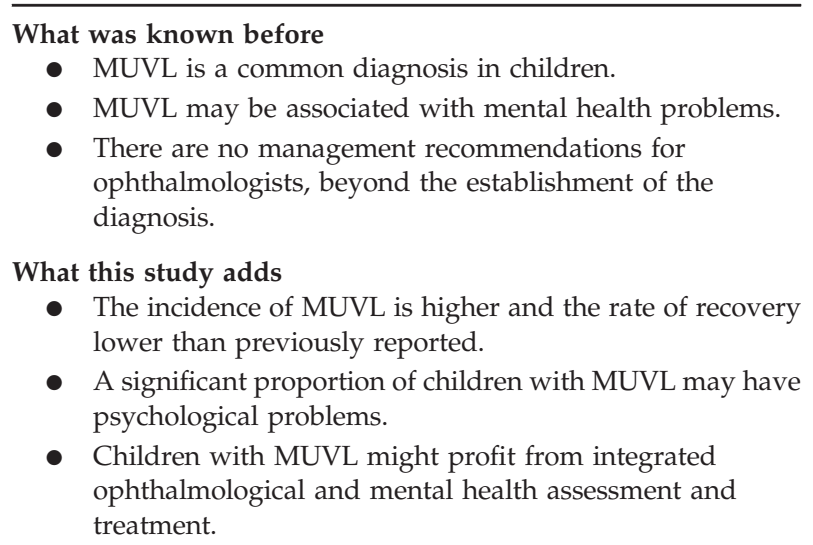

\section{Conflict of interest}

The authors declare no conflict of interest.

\section{Acknowledgements}

The study was not supported by specific funding. AD-N and MCD are employed by the National Institute for Health Research (NIHR) Moorfields Biomedical Research Centre, and as such the work was supported by the NIHR. The views expressed are those of the authors and not necessarily those of the NHS, the NIHR or the Department of Health. The research was supported by the National Institute for Health Research Biomedical Research Centre at Great Ormond Street Hospital for Children NHS Foundation Trust and University College London. We thank the nurses at the children's emergency department at Moorfields Eye Hospital for supporting this study.

\section{References}

1 Edwards TM, Stern A, Clarke DD, Ivbijaro G, Kasney LM. The treatment of patients with medically unexplained symptoms in primary care: a review of the literature. Ment Health Fam Med 2010; 7(4): 209-221.

2 Kinori M, Wygnanski-Jaffe T, Huna-Baron R. Functional visual loss in an Israeli pediatric population. Isr Med Assoc J 2011; 13(11): 684-688.

3 Middleton EM, Sinason MD, Davids Z. Blurred vision due to psychosocial difficulties: a case series. Eye 2008; 22(2): 316-317.

4 Gilbert A. Non-organic visual loss in children, 2015. Available at: http://www.aao.org/pediatric-center-detail/ neuro-ophthalmology-non-organic-visual-loss-in-chi (accessed on 28 October 2016).
5 Egan RA. Functional visual loss. Ophthalmol Clin North Am 2004; 17(3): 321-328, vi.

6 Eames TH. A study of tubular and spiral central fields in hysteria. Am J Ophthalmol 1947; 30(5): 610.

7 Kathol RG, Cox TA, Corbett JJ, Thompson HS, Clancy J. Functional visual loss: I. A true psychiatric disorder? Psychol Med 1983; 13(2): 307-314.

8 Beatty S. Non-organic visual loss. Postgrad Med J 1999; 75(882): 201-207.

9 Bain KE, Beatty S, Lloyd C. Non-organic visual loss in children. Eye 2000; 14(Pt 5): 770-772.

10 Mantyjarvi MI. The amblyopic schoolgirl syndrome. J Pediatr Ophthalmol Strabismus 1981; 18(6): 30-33.

11 (No authors listed) What to do about medically unexplained symptoms. Drug Ther Bull 2001; 39(1): 5-8.

12 Von Hiller W. [Somatization - conversion - dissociation: strategies for behavior therapy]. Z Psychosom Med Psychother 2005; 51(1): 4-22.

13 Lim SA, Siatkowski RM, Farris BK. Functional visual loss in adults and children patient characteristics, management, and outcomes. Ophthalmology 2005; 112(10): 1821-1828.

14 Toldo I, Pinello L, Suppiej A, Ermani M, Cermakova I, Zanin E et al. Nonorganic (psychogenic) visual loss in children: a retrospective series. J Neuroophthalmol 2010; 30(1): 26-30.

15 Taich A, Crowe S, Kosmorsky GS, Traboulsi EI. Prevalence of psychosocial disturbances in children with nonorganic visual loss. J AAPOS 2004; 8(5): 457-461.

16 Clarke WN, Noel LP, Bariciak M. Functional visual loss in children: a common problem with an easy solution. Can J Ophthalmol 1996; 31(6): 311-313.

17 Schlaegel TF Jr. Psychosomatic ophthalmology. Surv Ophthalmol 1956; 1(5): 411-421.

18 Wolpe ZS. Psychogenic visual disturbance in a four year old child. Nerv Child 1953; 10(2): 314-325.

19 Catalono RA, Simon JW, Krohel GB, Rosenberg PN. Functional visual loss in children. Ophthalmology 1986; 93(3): 385-390.

20 Scott JA, Egan RA. Prevalence of organic neuroophthalmologic disease in patients with functional visual loss. Am J Ophthalmol 2003; 135(5): 670-675.

21 Sabates NR, Gonce MA, Farris BK. Neuro-ophthalmological findings in closed head trauma. J Clin Neuroophthalmol 1991; 11(4): 273-277.

22 Keltner JL, May WN, Johnson CA, Post RB. The California syndrome. Functional visual complaints with potential economic impact. Ophthalmology 1985; 92(3): 427-435.

23 Vrabec TR, Levin AV, Nelson LB. Functional blinking in childhood. Pediatrics 1989; 83(6): 967-970.

24 Barris MC, Kaufman DI, Barberio D. Visual impairment in hysteria. Doc Ophthalmol 1992; 82(4): 369-382.

25 Geist R, Weinstein M, Walker L, Campo JV. Medically unexplained symptoms in young people: the doctor's dilemma. Paediatr Child Health 2008; 13(6): 487-491.

26 Masia Warner C, Reigada LC, Fisher PH, Saborsky AL, Benkov KJ. CBT for anxiety and associated somatic complaints in pediatric medical settings: an open pilot study. J Clin Psychol Med Settings 2009; 16(2): 169-177.

27 Kent C, McMillan G. A CBT-based approach to medically unexplained symptoms. Adv Psychiatr Treat 2009; 15(2): 146-151.

28 Husain K, Browne T, Chalder T. A review of psychological models and interventions for medically unexplained somatic symptoms in children. Child Adolesc Ment Health 2007; 12(1): $2-7$. 
29 Department of Health, London. Psychological Assessment and Treatment for Medically Unexplained Symptoms and Long-term Conditions. Department of Health: London, 2009.

30 Commissioning Support for London, NHS. Medically Unexplained Symptoms: A Whole Systems Approach. Commissioning Support for London: London, 2009.

31 McAllister E, Markham L, Coughtrey AE, Heyman I. Medically unexplained symptoms in children and adolescents. In: Skuse D, Bruce H, Dowdney L (eds). Child
Psychology and Psychiatry: Frameworks for Practice. Wiley: London, 2016.

32 McWilliams A, Reilly C, McFarlane FA, Booker E, Heyman I. Nonepileptic seizures in the pediatric population: a qualitative study of patient and family experiences. Epilepsy Behav 2016; 59: 128-136.

33 Reilly C, McWilliams A, Heyman I. What's in a name? 'Psychogenic' non-epileptic events in children and adolescents. Dev Med Child Neurol 2015; 57(1): 100-101. 